\title{
ESTRUCTURA Y COMPOSICIÓN FLORÍSTICA DE LA RESERVA FORESTAL - INSTITUCIÓN EDUCATIVA CAJETE, POPAYÁN (CAUCA)
}

\author{
Luis Eduardo López Vargas ${ }^{1,3}$ \\ Jorge Mario Becoche Mosquera, 3 \\ Diego Jesús Macías Pinto ${ }^{1,2,3}$ \\ Katherinn Ruiz Montoya ${ }^{1}$ \\ Alejandra Velasco Reyes ${ }^{1}$ \\ Soallin Pineda ${ }^{1}$
}

Recibido el 6 de agosto de 2014, aprobado el 22 de noviembre de 2014 y actualizado el 29 de abril de 2015

DOI: 10.17151/luaz.2015.41.8

\section{RESUMEN}

Se caracterizó la estructura y composición florística del remanente boscoso ubicado en la Reserva Forestal de la Institución Educativa Cajete, Popayán (Cauca). El inventario florístico se hizo mediante colecta libre realizada en el interior y la periferia del bosque. Se registraron en total 164 especies, 130 géneros y 58 familias. En Magnoliophyta se registraron 142 especies, 112 géneros y 44 familias; las familias más diversas fueron Asteraceae (31 especies y 26 géneros) y Araceae (10 especies y 3 géneros). En Lycophyta y Monylophyta se reconocieron 22 especies, 18 géneros y 14 familias; siendo Polypodiaceae con 4 especies la familia con mayor riqueza. Para determinar la estructura se muestrearon todos los individuos con DAP $\geq 1 \mathrm{~cm}$ en 10 bandas de $50 \times 2 \mathrm{~m}$, hallándose 560 individuos de plantas vasculares pertenecientes a 39 especies, 33 géneros y 25 familias. El bosque presentó 3 estratos: herbáceo, arbustivo y arbóreo. El estrato arbustivo fue el dominante con un elevado número de especies; el estrato arbóreo estuvo constituido por unas pocas especies. Quercus humboldtii y Banara guianensis fueron las especies con mayor dominancia e índice de valor de importancia en el bosque.

\section{PALABRAS CLAVE}

Estructura y composición florística, restauración, Cauca.

\section{FLORISTIC STRUCTURE AND COMPOSITION OF THE CAJETE EDUCATIONAL INSTITUTION FOREST RESERVE IN POPAYÁN (CAUCA).}

\begin{abstract}
The floristic structure and composition of the remaining wooded area located in the Cajete Educational institution forest reserve in Popayan (Cauca) was studied. The floristic inventory was performed through free collection carried out inside and in the periphery of the forest. In total 164 species, 130 genera and 58 families were recorded..In Magnoliophyta 142 species belonging
\end{abstract}


to 112 genera and 44 families were recorded, being Asteraceae (31 species and 26 genera) and Araceae (10 species and 3 genera) the most diverse families. In Monylophyta and Lycophyta 22 species belonging to 18 genera and 14 families were recognized, being Polypodiaceae with 4 species, the family with more richness. To determine the structure all individuals were sampled with $\geq 1 \mathrm{~cm}$ DAP in 10 bands of $50 \times 2 \mathrm{~m}$, and in total 560 individuals of vascular plants belonging to 39 species, 33 genera and 25 families were found. The forest presented three different strata: herbaceous, shrubby and arboreal. The shrubby stratum was dominant with a high number of species whereas the arboreal stratum consisted only of a few species. Quercus humboldtii and Banara guianensis were the species with greater dominance and with high importance value index in the forest.

\section{KEY WORDS}

floristic structure and composition, restoration, Cauca.

\section{INTRODUCCIÓN}

La diversidad biológica es fundamental para el desarrollo de planes de conservación y uso sostenible de los ecosistemas y sus componentes, por lo que su conocimiento, cuantificación y análisis, es esencial para entender la naturaleza y los cambios inducidos por la actividad humana (Villareal et al., 2004). Lo anterior ratifica el valor de los inventarios florísticos enfocados a responder las preguntas: ¿cuánta diversidad existe?, ¿dónde se encuentra? y ¿cómo se distribuye? Los estudios de composición florística, permiten conocer las especies de un área geográfica, su distribución y fisonomía (Escobar, 2013). También tienen impacto sobre la conservación del ambiente, porque se consigue una visión más amplia de los mecanismos biológicos que allí operan. Con ello se logran propagar las especies encontradas y preservar las condiciones ecológicas que permitan su existencia (Baquero et al., 2011).

Colombia presenta una gran riqueza florística representada en las regiones del Chocó biogeográfico y la Amazonía, sin embargo hay evidencia de que la mayor diversidad se concentra en el piedemonte y las estribaciones de las cordilleras (Alvear et al., 2010).

Diversos estudios han demostrado que la región andina es una de las más diversas de América tropical, favoreciendo la aparición de ambientes con características que brindan oportunidades excepcionales de adaptación y especiación (IAvH et al., 2010). Estos atributos permiten que los suelos sean aptos para actividades agropecuarias y agrícolas y los ecosistemas andinos estén sometidos a fuertes presiones que afectan la composición y fisonomía florística (Baquero et al., 2011). 
La estructura, composición y distribución de la flora caucana, comprendida entre 1700 y $2000 \mathrm{~m}$, presenta una dominancia de dos tipos de bosque: uno donde el roble (Quercus humboldtii) es dominante, y el otro donde esta especie es menguada y predomina cualquier otra según el grado de conservación o intervención de área. Además el dosel del bosque puede alcanzar hasta $35 \mathrm{~m}$ de altura, y en ellos es común la presencia de palmas y especies de las familias Lauraceae y Sapotaceae; así como variadas epífitas, orquídeas y helechos arbóreos (Bolaños et al., 2010).

Lastimosamente, durante el período 1985-2000, los ecosistemas naturales boscosos subandinos perdieron casi 400.000 ha, y de estas una considerable proporción corresponde a agroecosistemas, aunque también se registra un ligero aumento de ecosistemas asociados a coberturas del suelo en regeneración secundaria, cambio observado a lo largo de las tres cordilleras (Rudas et al., 2007). En esta contribución se da a conocer la composición y estructura florística del área de la Reserva Forestal de la Institución Educativa Cajete (RFIEC), ubicada en la meseta de Popayán, que puede ser utilizada para iniciar los procesos de restauración de los ecosistemas de este territorio.

\section{MÉTODO}

\section{Área de estudio}

La RFIEC se ubica en el municipio de Popayán, Cauca, entre los $2^{\circ} 28^{\prime} \mathrm{N}$ y $76^{\circ} 39 \mathrm{~W}$, con altitudes entre 1640 y $1730 \mathrm{~m}$ (Figura 1). La temperatura y la precipitación media anual son de $21^{\circ} \mathrm{C}$ y $1935 \mathrm{~mm}$ respectivamente. Según el sistema de zonas de vida el remanente de bosque se clasifica como húmedo premontano tropical (bh-P/T) (Holdridge, 1978) y selva subandina (Cuatrecasas, 1958). La reserva está rodeada al occidente por sembradíos de pancoger, al oriente por potreros y las instalaciones de la Institución Educativa (IE), al norte con el río Cauca y al sur con la vía que conduce al Tambo. Es uno de los pocos remanentes de bosque de la zona; posee un afloramiento de agua intermitente, a lo largo del área estudiada que desemboca en el río Cauca, razón clave para conservar el lugar por parte de la IE y garantizar el recurso hídrico como servicio ecosistémico de soporte para los pobladores (PNGIBSE, 2011). 


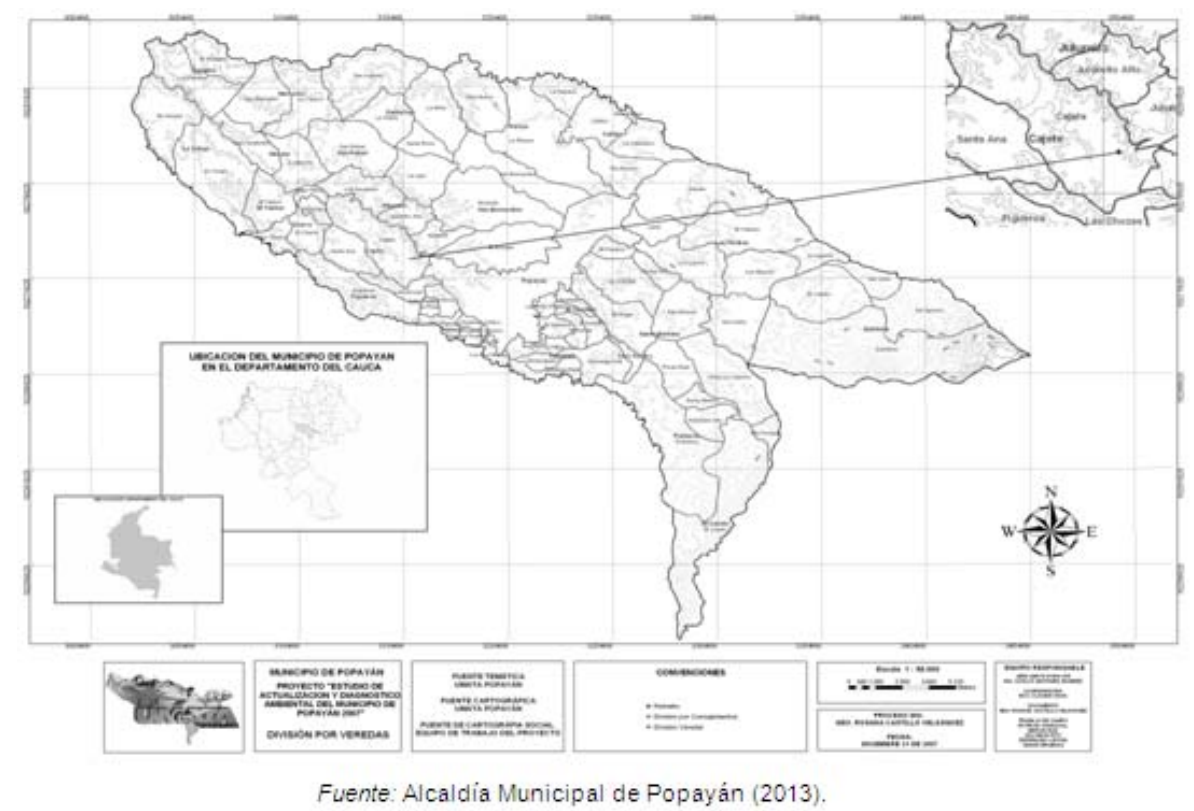

Figura 1. Ubicación geográfica y área de estudio.

\section{Composición y estructura}

Se realizaron salidas de campo entre enero y junio de 2012. El muestreo se realizó en toda el área del bosque (3,5 ha). Las muestras colectadas fueron procesadas siguiendo las técnicas estándar de herborización y se depositaron en el herbario de la Universidad del Cauca (CAUP). La determinación de especies se realizó utilizando claves especializadas (Mendoza y Ramírez, 2000; Giraldo et al., 2012) y las base de datos en línea de MOBOT y Plant List. Para el estudio de la estructura del área boscosa, se situaron 10 bandas de $50 \times 2 \mathrm{~m}(0,1 \mathrm{ha})$, evitando su traslape. En cada banda se censaron todos los individuos con $\mathrm{DAP} \geq 1 \mathrm{~cm}$, se anotó la especie, el perímetro a la altura del pecho (CAP), la altura del fuste y la altura total (m) (Álvarez et al., 2001).

\section{Tratamiento de la información}

Se elaboró un listado por familias y especies para determinar la riqueza de estas; con la información recopilada en los transectos se calcularon los siguientes parámetros e índices estructurales: densidad (D), densidad relativa (DR), frecuencia (F), frecuencia relativa (FR), dominancia (Do), dominancia relativa (DoR), índice de valor de importancia (IVI) y se halló el coeficiente de mezcla (CM).

Este análisis de la estructura horizontal cuantifica la participación de cada especie con relación a las demás y muestra cómo se distribuyen espacialmente. Este aspecto puede ser determinado por los anteriores índices que expresen la cantidad de árboles, su tamaño y distribución espacial (Acosta et al., 2006). 


\section{RESULTADOS Y DISCUSIÓN}

\section{Composición}

La flora vascular de la RFIEC presentó un total de 164 especies, correspondiente a 130 géneros y 58 familias.

En Magnoliophyta (Anexo 1), se registraron 142 especies distribuidas en 112 géneros y 44 familias (Figura 2). Las familias con más especies fueron Asteraceae con 31 especies, Araceae (10), Rubiaceae y Melastomataceae (8 especies cada una).

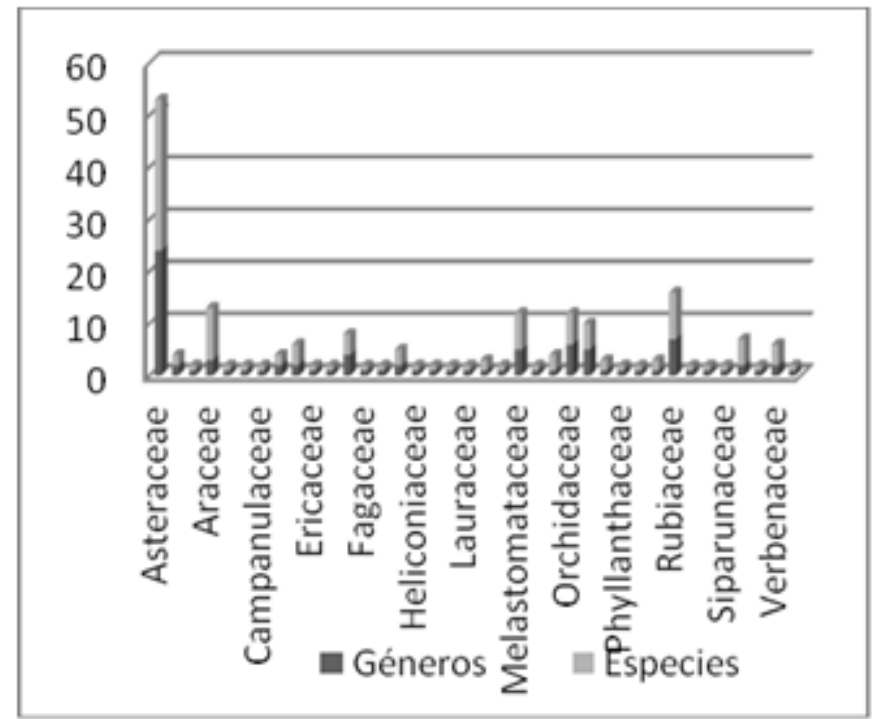

Figura 2. Relación Géneros y Especies de cada familia de Magnoliophytas en la RFIEC.

La mayoría de especies de Asteraceae, Melastomataceae y Myrtaceae se encontraron en zonas abiertas de la reserva, mientras que Piperaceae, Orchidaceae, Araceae y los helechos tanto epifitos como terrestres se hallaron en zonas con sombra, con excepción de Pteridium arachnoideum que está asociado a potreros y áreas abiertas.

En Lycophyta y Monilophyta (Anexo 2, Figura 3) se reconocieron 22 especies, 18 géneros y 14 familias. La familia con más especies fue Polypodiaceae con cuatro especies, seguida por Thelypteridaceae, Blechnaceae, Anemiaceae, Cyatheaceae y Gleicheniaceae con dos especies cada una. Las familias restantes presentaron una especie; los géneros con más especies fueron Blechnum, Serpocaulon y Anemia (2 especies). 


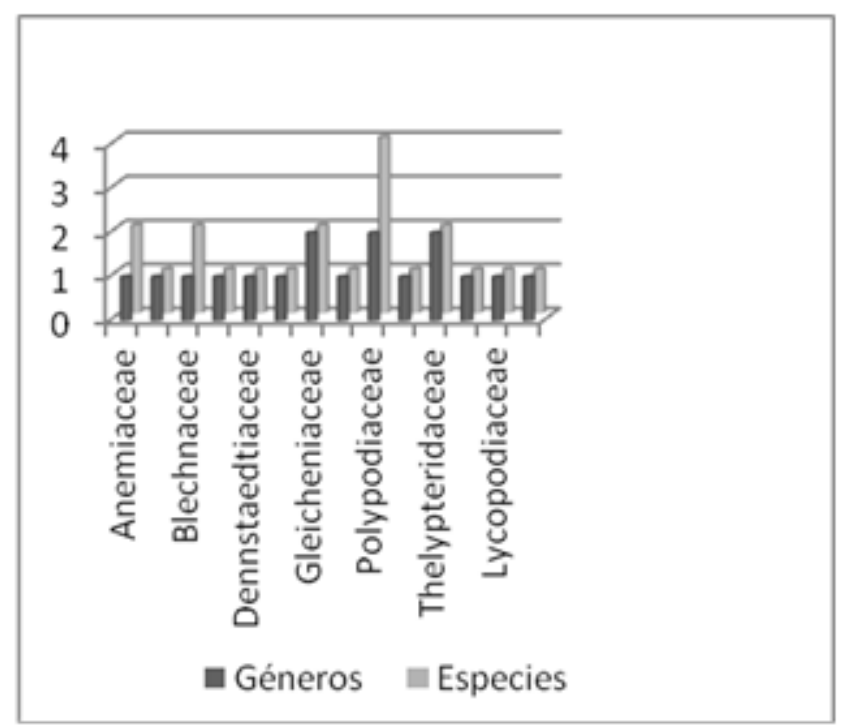

\section{Figura 3. Relación Familia, Género y Especie de Monilophytos y Licophytos de la RFIEC.}

Se puede observar que el relicto de bosque de la RFIEC, a pesar de su área reducida (3,5 ha), es muy parecido en estructura y composición a otros bosques de la planicie de Popayán, como el de La Rejoya (2,5 ha) (Bolaños et al., 2010). La familia Asteraceae fue la más diversificada en ambos estudios; el estrato arbustivo fue dominante y el arbóreo estuvo representado por pocas especies; por el contrario, se presentan diferencias con el estudio realizado en un bosque en las riberas del río Palacé, a 1920 m (Bolaños et al., 2002) que reporta menos especies y familias (33 y 14 respectivamente) a los encontrados en la RFIEC. Lo anterior es evidencia de la heterogeneidad de los remanentes de bosque de la planicie payanesa y la acción antrópica que además genera restricción a la dispersión y establecimiento de muchas especies como se refleja en la poca representatividad de especies de las familias Lauraceae y Ericaceae, que son una fuente importante de alimento para la fauna silvestre (Pincheira et al., 2009).

La presencia de las familias Rubiaceae y Melastomataceae, para este tipo de zonas de vida, son indicadoras de áreas de bosque secundario. De igual manera, de la abundancia de la familia Asteraceae y sus especies con preferencia de zonas abiertas y con buena iluminación, se puede inferir que el relicto boscoso RFIEC ha sufrido fuertes cambios generados por procesos antrópicos que han ocasionado pérdida de diversidad florística.

La importancia y representatividad en el área de estudio de la familia Araceae, es favorecida posiblemente por la quebrada intermitente presente, la cual genera ambientes húmedos, propicios para el desarrollo de las especies de esta familia (Cuatrecasas, 1958). 


\section{Estructura}

La estructura del bosque de la RFIEC muestra a Quercus humboldtii como la especie con mayor abundancia e IVI (Anexo 3), seguida de Banara guianensis. Los individuos de estas especies tienen en su mayoría DAP < $10 \mathrm{~cm}$ y unos pocos superan los $20 \mathrm{~cm}$ de DAP, lo que sumado a la dominancia del estrato arbustivo y de Guadua angustifolia representan un bosque multiestratificado en estado sucesional secundario.

Estructuralmente, se distinguen 3 estratos: herbáceo, arbustivo y arbóreo (Figura 4). El estrato herbáceo hasta los $1,5 \mathrm{~m}$ y conformado básicamente por: Anthurium longigeniculatum, $A$. sanguineum, Justicia chlorostachya, Chaptalia nutans, Calea sessiliflora, Sanicula liberta, Cuphea strigulosa, C. racemosa, Malaxis andicola, Sobralia violacea, Pseudechinolaena polystachya, Lantana camara y L. rugulosa.

El estrato arbustivo de 1,5 a $4 \mathrm{~m}$ de altura es el más común y dominante en número de especies, en el que se destacan: Toxicodendron acuminatum, Verbesina barragana, Desmodium adscendens, Mimosa albida, Vismia lauriformis, Tibouchina lindeniana, Siparuna aspera, Palicourea heterochroma, $P$. thyrsiflora, Psychotria ostreophora, Psidium guajava y $P$. guineense.

El estrato arbóreo representado por plantas entre 5 y $20 \mathrm{~m}$ de altura, está conformado por pocas especies y pocos individuos; entre estos sobresalen: Beilschmedia sp., Ocotea sp., Quercus humboldtii, Banara guianensis, Erythroxylum citrifolium, Syzygium jambos, Nectandra acutifolia, Cecropia angustifolia, Cupania americana, Guarea sp., Cinnanomun triplinerve y Cnemidaria horrida.

El epifitismo reportado para la meseta de Popayán es alto, debido a las condiciones de humedad de la planicie (Bolaños et al., 2010), sin embargo en la reserva se encontraron pocas especies y pocos individuos como Anthurium pedatum, Tillandsia fendleri, Epidendrum elongatum y Peperomia bilobulata, entre otras; posiblemente por la escasez arbórea, que restringe microclimas y soporte aptos para el desarrollo de este grupo de plantas. Tampoco representa el marcado predominio de las monocotiledóneas, especialmente de orquídeas sobre los demás grupos expresado para el neotrópico (Gentry y Dodson, 1987). 


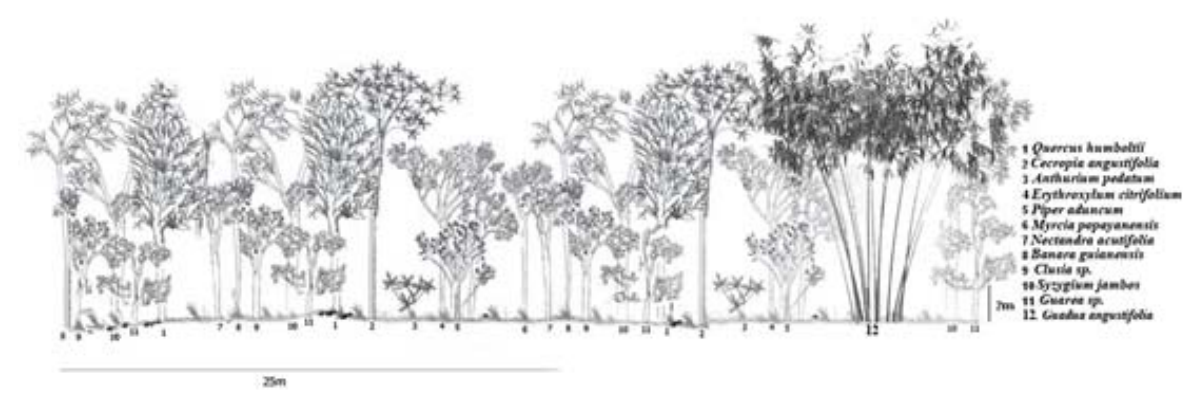

Figura 4. Perfil idealizado de la estructura vertical, del relicto boscoso de la RFIEC.

Las trepadoras, se encontraron especialmente en el borde del relicto boscoso, representada por pocas especies y pocos individuos de la familia Asteraceae: Oligactis volubilis, Critonia sp. y Mikania banisteriae.

En el área muestreada (0,1 ha), se registraron 560 individuos de plantas vasculares con un DAP $\geq 1 \mathrm{~cm}$, pertenecientes a 39 especies, distribuidas en 33 géneros y 25 familias. El número de especies fue equivalente al de otros reportes de ambientes con características similares (Gutiérrez y Rojas, 1996; Alcázar et al., 2002; Bolaños et al., 2010).

Lo anterior respalda la modificación metodología de Álvarez et al. (2001) a la de Gentry y Dodson (1987), que censa los individuos con DAP $\geq 1 \mathrm{~cm}$ (antes $\geq 2,54$ ) que permite registrar mayor número de especies en estos ecosistemas (Tabla 1).

Tabla 1. Registros con DAP $\geq 1 \mathrm{~cm}$ y DAP $\geq 2,54 \mathrm{~cm}$

\begin{tabular}{|l|c|c|c|c|}
\hline & Familias & Géneros & Especies & Individuos \\
\hline DAP $\geq 1$ & 25 & 33 & 39 & 560 \\
\hline DAP $\geq 2,54$ & 25 & 32 & 28 & 385 \\
\hline$\neq$ & 0 & 1 & 11 & 175 \\
\hline
\end{tabular}

Las especies con densidad relativa más alta (Figura 5) fueron Quercus humboldtii (15\%), seguido de Banara guianensis (11\%). Las familias mejor representadas, en cuanto número de individuos son: Fagaceae con 84, Myrtaceae con 73, Salicaceae con 63 y Lauraceae con 55; que representan el $48 \%$ del total de individuos. 


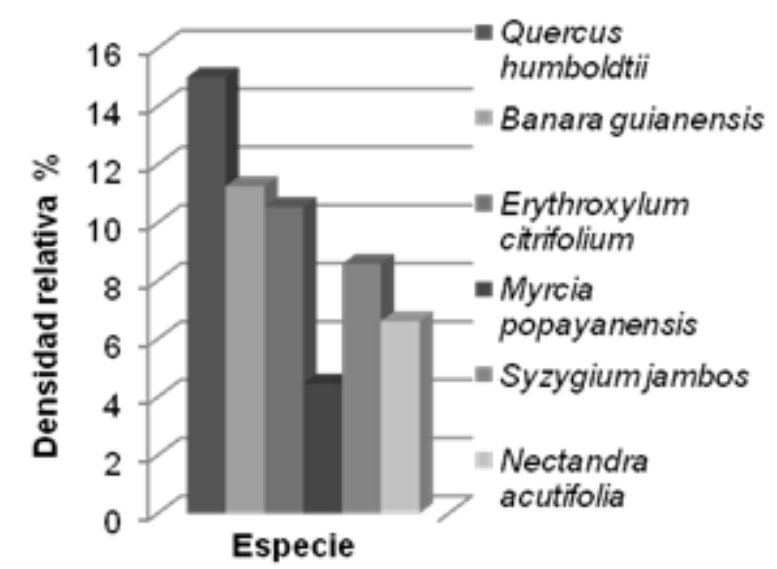

Figura 5. Especies con valores de densidad relativa más altos en la RFIEC.

Las especies más frecuentes fueron Quercus humboldtii, seguido de Banara guianensis, Syzygium jambos, Erythroxylum citrifolium, Myrcia popayanensis y Cecropia angustifolia (Anexo $3)$.

Los DAP en la mayoría de los individuos, estuvieron por debajo de $15 \mathrm{~cm}$, mientras que los valores por encima de $20 \mathrm{~cm}$ estuvieron representados por muy pocos individuos. Esto indica que la vegetación se encuentra en estadios tempranos de desarrollo.

En los valores de dominancia y dominancia relativa (Anexo 3), las especies más representativas fueron: Q. humboldtii, $B$. guianensis y $M$. popayanensis (Figura 6). Estas especies producen frutos con pericarpios suculentos que representan una buena fuente de alimentación para el gremio de frugívoros presentes en el área (Correa, 2013) y permiten la dispersión de las diásporas a lugares cercanos, favoreciendo la regeneración natural y el mantenimiento de la biodiversidad.

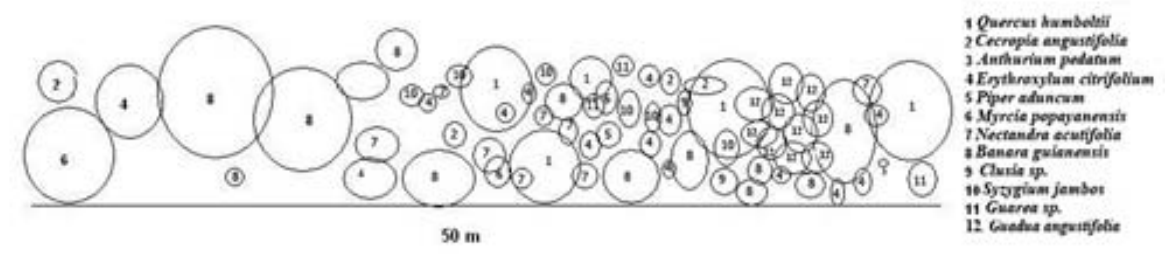

Figura 6. Perfil idealizado de la estructura horizontal, del relicto boscoso de la RFIEC.

Las especies con mayor peso ecológico (IVI), presentes en la RFIEC fueron: Quercus humboldtii $(36,7)$, Banara guianensis $(31,8)$, Erythroxylum citrifolium $(22,2)$ y Myrcia popayanensis $(21,2)$, difieren de las presentes en otros relictos similares como La Rejoya (Bolaños et al., 2010), donde el IVI mayor lo obtuvieron Alchornea latifolia $(96,3)$, Syzygium jambos $(42,2)$, Lacistema aggregatum $(29,6)$, Palicourea heterochroma $(18,4)$ y Hedyosmum bonplandianum $(17,6)$, que representa la 
heterogeneidad de los bosques que componen esta zona de vida.

El coeficiente de mezcla $(1: 14,35)$ de este bosque difiere significativamente de otro bosque dominado por roble en la planicie payanesa que reporta un CM de 1: 42,5 (Alvis, 2009); confirmando su tendencia a la heterogeneidad a pesar de la fuerte intervención antrópica. El relicto boscoso presenta características fisonómicas importantes como su cercanía a la cuenca del río Cauca y la presencia de un afluente, que permite el desarrollo funcional del ecosistema, sin embargo se requieren acciones para la restauración de áreas aledañas al remanente y ejecutar un plan de acción que involucre a la comunidad educativa de la Institución para garantizar su conservación, a través del reconocimiento de este espacio como aula viva.

Las actividades antrópicas (cultivos, entresaca de madera y pastoreo de ganado), siguen presionando el área del bosque, lo que ha ocasionado el asilvestramiento de especies foráneas, como Syzygium jambos.

Como otros remanentes, este representa una oportunidad de subsistencia de la flora regional y la fauna asociada a esta y se convierte en un banco de semillas que puede emplearse en futuros programas de restauración ecológica (Barrera et al., 2010).

\section{CONCLUSIONES}

En la RFIEC se hallaron 164 especies, equivalentes al 15\% de la flora vascular estimada para el municipio de Popayán. El mayor número de especies lo aportan las familias Asteraceae y Araceae, los cuales son taxones característicos de bosques perturbados y de áreas húmedas, respectivamente.

Los resultados permiten concluir que el remanente boscoso de la RFIEC, corresponde a un bosque natural en estado de regeneración secundaria.

Las condiciones topográficas del área de estudio como son las pendientes pronunciadas, la influencia de la cuenca media del río Cauca, la quebrada intermitente y el hecho de estar dentro de una Institución Educativa han favorecido el mantenimiento del relicto de bosque y se genera un potencial para restaurar áreas aledañas con participación de la comunidad.

\section{AGRADECIMIENTOS}

Los autores expresan su agradecimiento a las siguientes entidades y personas: Universidad del Cauca, Herbario Universidad del Cauca (CAUP), Institución Educativa Cajete, por 
su contribución para la realización del estudio; al profesor Pablo Gamboa por su acompañamiento en campo, y a los evaluadores por sus sugerencias y aportes.

\section{POTENCIAL CONFLICTO DE INTERESES}

Ninguno.

\section{FUENTES DE FINANCIACIÓN}

Universidad del Cauca.

\section{REFERENCIAS}

- Acosta, V.H., Araújo, P.A. e Iturre, M.C. (2006). Caracteres estructurales de las masas. Facultad de Ciencias Forestales. Universidad Nacional Santiago del Estero.

- Alcaldía Municipal de Popayán. (2013). Mapas territoriales. Recuperado de www.popayancauca.gov.co/nuestromunicipio.shtml?apc $=\mathrm{mm}$ xx1-\&x=1364653

- Alcázar, C., Díaz, S., Salgado, B. y Ramírez, B. (2002). Estructura y composición de un remanente de bosque subandino, Popayán. En Freire, F. y Neill, D. (Comps.), Memorias III Congreso Ecuatoriano de Botánica (pp. 163-180). Quito: Fundación Ecuatoriana para la Investigación y el Desarrollo de la Botánica.

- Álvarez, E, Cogollo, A, Rincón, H., Benítez, D., Parra, T., Rodríguez, W., Idárraga, A., Velásquez, C. y Jiménez, E. (2001). Propuesta metodológica de parcelas normatizadas para los inventarios de vegetación ISA-JAUM Documento de trabajo. Versión 1.7. Medellín, Colombia.

- Alvear, M., Betancur, J. y Franco, P. (2010). Diversidad florística y estructura de remanentes de bosque andino en la zona de amortiguación del Parque Nacional Natural los Nevados, Cordillera Central Colombiana. Caldasia, 32(1), 39-63.

- Alvis, J. (2009). Análisis estructural de un bosque natural localizado en zona rural del municipio de Popayán. Biotecnología en el sector agropecuario, 7(1), 115-122.

- Baquero, J., Gómez, G. y Orozco, J. (2011). Composición florística en la sonadora, Calarcá, Quindío. Armenia: Centro de Estudios e Investigaciones en Biodiversidad y Biotecnología CIBUQ, Universidad del Quindío.

- Barrera, J., Contreras, S., Garzón, N., Moreno, A. y Montoya, S. (2010). Manual para la Restauración Ecológica de los Ecosistemas Disturbados del Distrito Capital. Bogotá: Secretaría Distrital de Ambiente (SDA) y Pontificia Universidad Javeriana (PUJ).

- Bolaños, G., Chito, E. y Feuillet, C. (2002). Inventario florístico de un remanente de bosque del municipio de Popayán, Cauca, 
Colombia. En Rangel, O., Aguirre, J. y Andrade, G. (Comps.), Memorias VIII Congreso Latinoamericano y II Colombiano de Botánica (pp. 411-412). Bogotá: Universidad Nacional de Colombia.

- Bolaños, G., Feuillet, C., Chito, E., Muñoz, E. y Ramírez, B. (2010). Vegetación, estructura y composición de un área boscosa en el Jardín Botánico "Álvaro José Negret", Vereda La Rejoya, Popayán (Cauca, Colombia). Boletín Científico Centro de Museos Museo de Historia Natural Universidad de Caldas, 14(2), 19-38.

- Correa, D. (2013). Patrones de Frecuencia y Abundancia de Sistemas de Dispersión de Plantas en Bosques Colombianos y su Relación con las Regiones Geográficas del País. Colombia Forestal, 16(1), 33-51.

- Cuatrecasas, J. (1958). Aspectos de la vegetación natural de Colombia. Revista de la Academia Colombiana de Ciencias Exactas, Físicas y Naturales, 10(40), 221-268.

- Escobar, N. (2013). Diagnóstico de la Composición Florística Asociada a Actividades Agropecuarias en el Cerro Quinini (Colombia). Revista Ciencias Agropecuarias. Universidad de Cundinamarca, 1(1), 10-28.

- Gentry, A. y Dodson, C. (1987). Diversity and Biogeography of Neotropical Vascular Epiphytes. Annals of the Missouri Botanical Garden, 74(2), 205-233.

- Giraldo, D., Peterson, P. y Sánchez, I. (2012). The genus Eragrostis (Poaceae: Chloridoideae) in northwestern South America (Colombia, Ecuador, and Peru): Morphological and taxonomic studies. Biblioteca José Jerónimo Triana, 24, 1-195.

- Gutiérrez, V. y Rojas, A. (1996). Comparación de dos remanentes de bosque de la finca Los Robles, municipio de Timbío, departamento del Cauca. Trabajo de Grado Ecología. Fac. Ciencias Naturales, Fundación Universitaria de Popayán. Popayán.

- Holdridge, L. (1978). Ecología basada en zonas de vida. (2a ed). Costa Rica: Serie libros y materiales educativos.

- IAvH, IDEAM, IIAP, INVEMAR y SINCHI. (2010). Informe del estado del medio ambiente y de los recursos naturales renovables. Instituto de hidrología, meteorología y estudios ambientales. Bogotá: IDEAM.

- Mendoza, H. y Ramírez, B. (2000). Plantas y Flores de La Planada, Guía ilustrada de familias y géneros. Bogotá: Panamericana.

- Pincheira, J., Rau, J. y Cortez, F. (2009). Tamaño y forma de fragmentos de bosque y su relación con la riqueza de especies de árboles y arbustos. FYTON, 78, 121-128.

- Política Nacional para la Gestión Integral de la Biodiversidad y sus Servicios Ecosistémicos (PNGIBSE). (2011). Bogotá: Instituto Alexander von Humboldt.

- Rudas, G., Marcelo, D., Armenteras, D., Rodríguez, N., Morales, M., Delgado, L. y Sarmiento, A. (2007). Biodiversidad y actividad humana: relaciones en ecosistemas de bosque subandino en Colombia. Bogotá: Instituto Alexander von Humboldt

- Villarreal, H., Álvarez, M., Córdoba, S., Escobar, F., Fagua, G. y Gast, F. (2004). Manual de métodos para el desarrollo de inventarios de biodiversidad. Programa de inventarios de biodiversidad. Bogotá: Instituto Alexander von Humboldt. 
1. Programa de Biología. Facultad de Ciencias Naturales Exactas y de la Educación, Universidad del Cauca. Popayán, Colombia.

2. Profesor Universidad del Cauca.

3. Grupo de Estudios sobre Diversidad Vegetal (Sachawaira). Correspondencia (respectivamente): lelopez@unicauca.edu.co; jbecoche@unicauca.edu.co; djmacias@unicauca.edu.co; lkruiz@unicauca.edu.co; alejavelasco@unicauca.edu.co; skpineda@unicauca.edu.co 
Anexo 1. Magnoliophythas registradas en la RFIEC

\begin{tabular}{|c|c|c|}
\hline Familia & Especie & Nro. en CAUP \\
\hline Acanthaceae & $\begin{array}{l}\text { Justicia chlorostachya Leonard } \\
\text { Thunbergia alata Bojer ex Sims } \\
\text { Trichantera gigantea (Humb. \& Bonpl.) Nees }\end{array}$ & $\begin{array}{l}\text { A. Velasco } 1 \\
\text { A. Velasco } 2 \\
\text { L. López } 125\end{array}$ \\
\hline Anacardiaceae & $\begin{array}{l}\text { Mauria heterophylla Kunth } \\
\text { Toxicodendron acuminatum (DC.) C.Y. Wu \& T.L. Ming }\end{array}$ & $\begin{array}{l}\text { L. Lopez } 126 \\
\text { K. Ruiz } 1\end{array}$ \\
\hline Araceae & $\begin{array}{l}\text { Anthurium longigeniculatum Engl. } \\
\text { Anthurium martae Croat \& Castaño Rubiano } \\
\text { Anthurium nigrenscens Engl. } \\
\text { Anthurium pedatum Endl. ex Kunth } \\
\text { Anthurium sanguineum Engl. } \\
\text { Anthurium formosum Schott } \\
\text { Anthurium } \mathrm{sp} .1 \\
\text { Anthurium } \mathrm{sp} .2 \\
\text { Monstera punctulata (Schott) Schott ex Engl. } \\
\text { Philodendron } \mathrm{sp} \text {. }\end{array}$ & $\begin{array}{l}\text { S.Pineda } 16 \\
\text { S. Pineda } 15 \\
\text { S. Pineda } 14 \\
\text { S. Pineda } 13 \\
\text { S. Pineda } 17 \\
\text { S. Pineda } 12 \\
\text { S. Pineda } 19 \\
\text { S.Pineda } 21 \\
\text { S. Pineda } 18 \\
\text { S.Pineda } 20\end{array}$ \\
\hline Asteraceae & $\begin{array}{l}\text { Acmella ciliata (Kunth) Cass. } \\
\text { Ageratum conyzoides (L.) L. } \\
\text { Austroeupatorium inulifolium (Kunth) R.M. King \& H. } \\
\text { Rob. } \\
\text { Baccharis decusata (Klatt.) Hieron } \\
\text { Baccharis pedunculata (Mill.) Cabrera } \\
\text { Baccharis trinervis (Lam.) Pres. } \\
\text { Bidens pilosa L. } \\
\text { Calea sessiliflora Less. } \\
\text { Chaptalia nutans (L.) Pol. } \\
\text { Chromolaena laevigata (Lam.) R.M. King \& H. Rob. }\end{array}$ & $\begin{array}{l}\text { L. López } 127 \\
\text { L. López } 128 \\
\text { L. López } 129 \\
\text { L. López } 130 \\
\text { L. López } 131 \\
\text { L. López } 132 \\
\text { L. López } 133 \\
\text { L. López } 134 \\
\text { L. López } 135 \\
\text { L. López } 136 \\
\text { L. López } 137\end{array}$ \\
\hline
\end{tabular}




\begin{tabular}{|c|c|c|}
\hline & $\begin{array}{l}\text { Chromolaena tacotana (Klatt) R.M. King \& H. Rob. } \\
\text { Condylopodium cuatrecasaii R.M. King y H. Rob. } \\
\text { Conyza bonariensis (L.) Cronquist } \\
\text { Crepis japonica (L.) Benth } \\
\text { Critonia sp. } \\
\text { Elaphandra lehmannii (Hieron) Pruski } \\
\text { Elaphandra sp. } \\
\text { Elephantopus mollis Kunth } \\
\text { Emilia coccinea (Sims) G. Don } \\
\text { Gamochaeta americana (Mill.) Wedd. } \\
\text { Hypochaeris radicata L. } \\
\text { Mikania banisteriae } \\
\text { Munnozia hastifolia (Poepp.) H. Rob. \& Brettell } \\
\text { Oligactis volubilis (Kunth) Cass. } \\
\text { Siegesbeckia sp. } \\
\text { Sonchus oleraceus L. } \\
\text { Sonchus asper (L.) Hill } \\
\text { Tagetes caracasana Humb. ex Willd } \\
\text { Tithonia diversifolia (Hemsl.) A. Gray } \\
\text { Verbesina barragana Cuatrec. } \\
\text { Wedelia sp. }\end{array}$ & $\begin{array}{l}\text { L. López } 138 \\
\text { L. López } 139 \\
\text { L. López } 140 \\
\text { L. López } 141 \\
\text { L. López } 142 \\
\text { L. López } 143 \\
\text { L. López } 144 \\
\text { L. López } 145 \\
\text { L. López } 146 \\
\text { L. López } 147 \\
\text { L. López } 148 \\
\text { L. López } 149 \\
\text { L. López } 150 \\
\text { L. López } 151 \\
\text { L. López } 152 \\
\text { L. López } 153 \\
\text { L. López } 154 \\
\text { L. López } 155 \\
\text { L. López } 156 \\
\text { L. López } 157 \\
\text { L. López } 169\end{array}$ \\
\hline Apiaceae & Sanicula liberta Cham. \& Schtdl. & L. Lopez 158 \\
\hline Apiaceae & Sanicula liberta Cham. \& Schtdl. & L. Lopez 158 \\
\hline Arecaceae & Geonoma jussieuana Mart. & L. Lopez 159 \\
\hline Bromeliaceae & Tillandsia fendleri Griseb & L. Lopez 160 \\
\hline Campanulaceae & Centropogon sp. & L. Lopez 161 \\
\hline Commelinaceae & $\begin{array}{l}\text { Commelina robusta Kunth } \\
\text { Callisia gracilis (Kunth) D.R. Hunt }\end{array}$ & $\begin{array}{l}\text { S. Pineda } 10 \\
\text { S. Pineda } 9\end{array}$ \\
\hline Clusiaceae & clusia sp. & S. Pineda 29 \\
\hline Cyperaceae & $\begin{array}{l}\text { Rhynchospora corymbosa (L) Britton } \\
\text { Rhynchospora rugosa (Vahl) Gale } \\
\text { Rhyncospora nervosa (Vahl) Boecheler } \\
\text { Fimbristylis dichotoma (L.) Vahl }\end{array}$ & $\begin{array}{l}\text { A. Velasco } 3 \\
\text { A. Velasco } 4 \\
\text { A. Velasco } 23 \\
\text { L. López } 162\end{array}$ \\
\hline Ericaceae & Monotropa unifiora $L$. & K. Ruiz 29 \\
\hline Erythroxylaceae & Erythroxylum citrifolium A. St.-Hill. & Avelasco 26 \\
\hline Euphorbiaceae & Acalypha macrostachya Jacq & K. Ruiz 2 \\
\hline Fabaceae & $\begin{array}{l}\text { Centrosema virginianum (L.) Benth } \\
\text { Desmodium adscendens (Sw.) DC. } \\
\text { Inga densiflora Benth } \\
\text { Mimosa albida H. \& Bonpl ex Willd }\end{array}$ & $\begin{array}{l}\text { S. Pineda } 7 \\
\text { L. López } 163 \\
\text { L. López } 164 \\
\text { S. Pineda } 8\end{array}$ \\
\hline Fagaceae & Quercus humboldtil Bonpl & K. Ruiz 3 \\
\hline Gentianaceae & Iribachia alata (AubI.) Maas & J. Becoche 22 \\
\hline Gesneriaceae & $\begin{array}{l}\text { Besleria solanoides Kunth } \\
\text { Kohleria inaequalis (Benth.) Wiehler } \\
\text { Kohleria spicata (Kunth) Oerst. }\end{array}$ & $\begin{array}{l}\text { J. Becoche } 23 \\
\text { A. Velasco } 18 \\
\text { A. Velasco } 17\end{array}$ \\
\hline
\end{tabular}




\begin{tabular}{|c|c|c|}
\hline Fabaceae & $\begin{array}{l}\text { Centrosema virginianum (L.) Benth } \\
\text { Desmodium adscendens (Sw.) DC. } \\
\text { Inga densiflora Benth } \\
\text { Mimosa albida H. \& Bonpl ex Willd }\end{array}$ & $\begin{array}{l}\text { S. Pineda } 7 \\
\text { L. López } 163 \\
\text { L. López } 164 \\
\text { S. Pineda } 8\end{array}$ \\
\hline Fagaceae & Quercus humboldtil Bonpl & K. Ruiz 3 \\
\hline Gentianaceae & Iribachia alata (AubI.) Maas & J.Becoche 22 \\
\hline Gesneriaceae & $\begin{array}{l}\text { Besleria solanoides Kunth } \\
\text { Kohleria inaequalis (Benth.) Wiehler } \\
\text { Kohleria spicata (Kunth) Oerst. }\end{array}$ & $\begin{array}{l}\text { J. Becoche } 23 \\
\text { A. Velasco } 18 \\
\text { A. Velasco } 17\end{array}$ \\
\hline Heliconiaceae & Heliconia sp. & L. Lopez 165 \\
\hline Hypericaceae & Vismia lauriformis (Lam.) Choisy & K. Ruiz 4 \\
\hline Lamiaceae & $\begin{array}{l}\text { Hyptis atrorubens Point } \\
\text { Scutellaria incarnata Vahl. }\end{array}$ & $\begin{array}{l}\text { A. Velasco } 6 \\
\text { A. Velasco } 5\end{array}$ \\
\hline Lauraceae & $\begin{array}{l}\text { Bleischmieda costaricensis (Mez \& Pittier) C.K. Allen } \\
\text { Cinnanomun triplinerve } \\
\text { Nectandra acutifolia (Ruiz \& Pav.) Mez } \\
\text { Ocotea sp. }\end{array}$ & $\begin{array}{l}\text { S. Pineda } 26 \\
\text { S. Pineda } 27 \\
\text { J. Becoche } 26 \\
\text { A. Velasco } 24\end{array}$ \\
\hline \begin{tabular}{|l} 
Lythraceae \\
\end{tabular} & $\begin{array}{l}\text { Cuphea racemosa (L.f.) Spreng. } \\
\text { Cuphea strigulosa Kunth }\end{array}$ & $\begin{array}{l}\text { J. Becoche } 24 \\
\text { J. Becoche } 25\end{array}$ \\
\hline Malvaceae & $\begin{array}{l}\text { Sida jamaicensis Cav. } \\
\text { Triumfetta lappula L. }\end{array}$ & $\begin{array}{l}\text { A. Velasco } 8 \\
\text { A. Velasco } 7\end{array}$ \\
\hline Melastomataceae & $\begin{array}{l}\text { Acinodendron grandiflorum (Cogn.) Kuntze } \\
\text { Clidemia ciliata Pav. ex D. Don } \\
\text { Clidemia sericea D. Don } \\
\text { Meriania speciosa (Bonpl.) Naudin } \\
\text { Miconia albicans (Sw.) Steud. } \\
\text { Miconia sp. } \\
\text { Tibouchina lindeniana Cogn. }\end{array}$ & $\begin{array}{l}\text { J. Becoche } 50 \\
\text { J. Becoche } 30 \\
\text { J. Becoche } 29 \\
\text { J. Becoche } 31 \\
\text { J. Becoche } 34 \\
\text { J. Becoche } 33 \\
\text { J. Becoche } 32\end{array}$ \\
\hline
\end{tabular}




\begin{tabular}{|c|c|c|}
\hline & Tibouchina gracilis (Bonpl.) Cogn & J. Becoche 35 \\
\hline Meliaceae & Guarea sp. & A. Velasco 25 \\
\hline Monimiaceae & Mollinedia sp. & S. Pineda 28 \\
\hline Myrtaceae & Myrcia popayanensis Hieron. & J. Becoche 48 \\
\hline & $\begin{array}{l}\text { Psidium guajava L. } \\
\text { Psidium guineense Sw. } \\
\text { Myrtaceae sp. } 1 \\
\text { Syzygium jambos (L.) Alston }\end{array}$ & $\begin{array}{l}\text { J. Becoche } 37 \\
\text { J. Becoche } 38 \\
\text { J. Becoche } 39 \\
\text { J. Becoche } 49\end{array}$ \\
\hline Orchidaceae & $\begin{array}{l}\text { Crocodeilanthe sp. } \\
\text { Elleanthus sp. } \\
\text { Epidendrum elongatum Jacq } \\
\text { Lepanthes sp. } \\
\text { Malaxis andicola (Ridl) Kuntze } \\
\text { Maxillaria sp. } \\
\text { Erycina pumilio (Rchb. f.) N.H. Williams \& M.W. Chase } \\
\text { Sobralia violacea Lindl. ex Lindl. }\end{array}$ & $\begin{array}{l}\text { A. Velasco } 20 \\
\text { S. Pineda } 11 \\
\text { J. Becoche } 42 \\
\text { A. Velasco } 19 \\
\text { J. Becoche } 40 \\
\text { L. Ruiz } 30 \\
\text { J. Becoche } 43 \\
\text { J. Becoche } 41\end{array}$ \\
\hline Piperaceae & $\begin{array}{l}\text { Peperomia bilobulata C. DC } \\
\text { Piper aduncum L. } \\
\text { Piper sp. }\end{array}$ & $\begin{array}{l}\text { A. Velasco } 21 \\
\text { L. López } 166 \\
\text { A. Velasco } 22\end{array}$ \\
\hline Phyllanthaceae & Phyllanthus niruri $\mathrm{L}$. & K. Ruiz 5 \\
\hline Plantaginaceae & Plantago australis Lam & A. Velasco 9 \\
\hline Poaceae & $\begin{array}{l}\text { Andropogum sp. } \\
\text { Axonopus compresus (Sw.) P. Beauv. } \\
\text { Guadua angustifolia L. } \\
\text { Panicum sp. } \\
\text { Pseudechinolaena polystachya (Kunth) Stapf. }\end{array}$ & $\begin{array}{l}\text { S. Pineda } 21 \\
\text { S. Pineda } 22 \\
\text { L. López } 167 \\
\text { S. Pineda } 23 \\
\text { S. Pineda } 24\end{array}$ \\
\hline
\end{tabular}




\begin{tabular}{|c|c|c|}
\hline & Sporobolus sp. & S. Pineda 25 \\
\hline Primulaceae & $\begin{array}{l}\text { Myrsine coriacea (Sw.)R.Br. ex Roem. \& Schult. } \\
\text { Myrsine guianensis (Aubl.) Kuntze }\end{array}$ & $\begin{array}{l}\text { S. Pineda } 28 \\
\text { J. Becoche } 36\end{array}$ \\
\hline Rosaceae & $\begin{array}{l}\text { Rubus rosifolios Sm. ex Baker } \\
\text { Rubus urticifolius Poir. }\end{array}$ & $\begin{array}{l}\text { J. Becoche } 45 \\
\text { J. Becoche } 44\end{array}$ \\
\hline Rubiaceae & $\begin{array}{l}\text { Coccocypselum lanceolatum (Ruiz \& Pav.) Pers } \\
\text { Coffea arabica L. } \\
\text { Galium hypocarpium (L.) Endl. Ex Griseb. } \\
\text { Palicourea heterochroma K. Schum. \& K. Krause } \\
\text { Psychotria ostreophora (Wernham) } \\
\text { Palicourea thyrsiffora (Ruiz \& Pav.) DC. } \\
\text { Psychotria sp. } \\
\text { Richardia scabra L. }\end{array}$ & $\begin{array}{l}\text { S. Pineda } 5 \\
\text { K. Ruiz } 7 \\
\text { K. Ruiz } 6 \\
\text { S. Pineda } 6 \\
\text { S. Pineda } 3 \\
\text { S. Pineda } 4 \\
\text { S. Pineda } 1 \\
\text { S. Pineda } 2\end{array}$ \\
\hline Salicaceae & Banara guianensis Aubl. & K. Ruiz 8 \\
\hline Sapindaceae & Cupania americana $\mathrm{L}$. & A. Velasco 27 \\
\hline Scrophulariacee & Castilleja scorzonerifolia Kunth. & K. Ruiz 9 \\
\hline Siparunaceae & Siparuna aspera (Ruiz \& Pav.)A.DC. & J. Becoche 46 \\
\hline Solanaceae & $\begin{array}{l}\text { Browallia americana } L \text {. } \\
\text { Browallia sp. } \\
\text { Solanum acerifolium Dunal. } \\
\text { Solanum asperolanatum Ducke } \\
\text { Solanum umbelliferum Eschsch. }\end{array}$ & $\begin{array}{l}\text { K. Ruiz } 31 \\
\text { A. Velasco } 12 \\
\text { A. Velasco } 10 \\
\text { A. Velasco } 11 \\
\text { J. Becoche } 51\end{array}$ \\
\hline Urticaceae & Cecropia angustifolia Trecul & L. Lopez 168 \\
\hline Caprifoliaceae & Astrephia chaerophylloides (Sm.) DC & J.Becoche 47 \\
\hline Verbenaceae & $\begin{array}{l}\text { Aloysia scorodonioides (Kunth) Cham. } \\
\text { Lantana camara L. } \\
\text { Lantana rugulosa Kunth. } \\
\text { Lantana trifolia L. }\end{array}$ & $\begin{array}{l}\text { A. Velasco } 16 \\
\text { A. Velasco } 13 \\
\text { A. Velasco } 14 \\
\text { A. Velasco } 15\end{array}$ \\
\hline
\end{tabular}


Anexo 2. Monilophytos y Lycophitos presentes en la RFIEC

\begin{tabular}{|c|c|c|}
\hline Familia & Especie & Nro. en CAUP \\
\hline Anemiaceae & $\begin{array}{l}\text { Anemia flexuosa (Savigny) Sw. } \\
\text { Anemia phyllitidis (L.) Sw. }\end{array}$ & $\begin{array}{l}\text { K. Ruiz } 10 \\
\text { K. Ruiz } 11\end{array}$ \\
\hline Aspleniaceae & Asplenium aethiopicum (Burm. F.) Bech. & K. Ruiz 12 \\
\hline Blechnaceae & $\begin{array}{l}\text { Blechnum cordatum (Desv.) Hieron. } \\
\text { Blechnum fraxinifolium Desv. }\end{array}$ & $\begin{array}{l}\text { K. Ruiz } 13 \\
\text { K. Ruiz } 14\end{array}$ \\
\hline Cyatheaceae & $\begin{array}{l}\text { Cnemidaria horrida (L. C. Presl.) } \\
\text { Cyathea sp. }\end{array}$ & $\begin{array}{l}\text { K. Ruiz } 16 \\
\text { K. Ruiz } 15\end{array}$ \\
\hline Dennstaedtiaceae & Pteridium arachnoideum (Kaulf.) Maxon & K. Ruiz 17 \\
\hline Lomariopsidaceae & Elaphoglossum cuspidatum (Wild.) Moore & K. Ruiz 18 \\
\hline Geicheniaceae & $\begin{array}{l}\text { Geichenella pectinata (Willd.) Ching } \\
\text { Sticherus bifidus (Willd.) Ching }\end{array}$ & $\begin{array}{l}\text { K. Ruiz 19 } \\
\text { K. Ruiz } 20\end{array}$ \\
\hline Hymenophyllaceae & Hymenophyllum sp. & K. Ruiz 21 \\
\hline Polypodiaceae & $\begin{array}{l}\text { Pecluma sp. } 1 \\
\text { Pecluma sp. } 2 \\
\text { Serpocaulon dissimile (L.) A.R. Sm } \\
\text { Serpocaulon triseriale (Sw.) A.R. Sm. }\end{array}$ & $\begin{array}{l}\text { K. Ruiz } 22 \\
\text { K. Ruiz } 32 \\
\text { K. Ruiz } 23 \\
\text { K. Ruiz } 24\end{array}$ \\
\hline Pteridaceae & Adiantum andicola Liebm. & K. Ruiz 25 \\
\hline Thelypteridaceae & $\begin{array}{l}\text { Macrothelypteris sp. } \\
\text { Thelypteris sp. }\end{array}$ & $\begin{array}{l}\text { K. Ruiz } 27 \\
\text { K. Ruiz } 26\end{array}$ \\
\hline Vittariaceae & Radiovittaria sp. & K. Ruiz 28 \\
\hline Lycopodiaceae & Huperzia reflexa (Lam.) Trevis & J. Becoche 27 \\
\hline Selaginellaceae & Selaginella geniculata (C. Presl.) Spring & J. Becoche 28 \\
\hline
\end{tabular}


Anexo 3. Variables fitosociológicas para plantas vasculares de la RFIEC

\begin{tabular}{|c|c|c|c|c|c|c|c|}
\hline Especie & Do & DoR & D & DR & $\mathrm{F}$ & FR & TVI \\
\hline Quercushumboldtii & 3394,10 & 13,57 & 840,00 & 15,00 & 0,90 & 8,18 & 36,75 \\
\hline Banara guianensis & 3321,62 & 13,28 & 630,00 & 11,25 & 0,80 & 7,27 & 31, \\
\hline Erythroxylum citrifolium & 1098,11 & 4,39 & 590,00 & 10,54 & 0,80 & 7,27 & 22,20 \\
\hline MyrC & 2602,85 & 10,41 & 250,00 & 4,46 & 0,70 & 6,36 & 1,2 \\
\hline Toxicodendron acuminatum & 4683,91 & 18,73 & 10,00 & 0,18 & 0,10 & 0,91 & 19,82 \\
\hline Syzygiumjambos & 74,02 & 0,30 & 480,00 & 8,57 & 0,80 & 7,27 & 16,14 \\
\hline Nectandra a & 232,20 & 0,93 & 370,00 & 6,61 & 0,60 & 5,45 & 12,99 \\
\hline Cecro, & 934,35 & 3,74 & 140,00 & 2,50 & 0,70 & 6,36 & 12,60 \\
\hline Guadua ang & 944,07 & 3,77 & 240,00 & 4,29 & 0,30 & 2,73 & 0,79 \\
\hline clusia sp. & 68,04 & 0,27 & 220,00 & 3,93 & 0,50 & 4,55 & 8,75 \\
\hline Myrs & 1631,99 & 6,53 & 20,00 & 0,36 & 0,20 & 1,82 & 8,70 \\
\hline Alcalypha macrostachia & 1270,66 & 5,08 & 90,00 & 1,61 & 0,10 & 0,91 & 7,60 \\
\hline Inga densifiora & 717,49 & 2,87 & 100,00 & 1,79 & 0,30 & 2,73 & 7,38 \\
\hline Mico & 85,34 & 0,34 & 190,00 & 3,39 & 0,40 & 3,64 & 7,37 \\
\hline Piperaduncum & 40,10 & 0,16 & 150,00 & 2,68 & 0,40 & 3,64 & 6,48 \\
\hline Pal & 18 & 76 & 200,00 & 3,57 & 0,20 & 1,82 & 6,15 \\
\hline Cup & 599,37 & 2,40 & 90,00 & 1,61 & 0,20 & 1,82 & 5,82 \\
\hline Coffea arabiga & 56,94 & 0,23 & 130,00 & 2,32 & 0,30 & 2,73 & 5,28 \\
\hline Cinnanomun & 36 & 0,46 & 140,00 & 2,50 & 0,20 & 1,82 & 4,78 \\
\hline Miconiagr & 102,56 & 0,41 & 100,00 & 1,79 & 0,20 & 1,82 & 4,01 \\
\hline Critoniasp. & 34,79 & 0,14 & 110,00 & 1,96 & 0,20 & 1,82 & 3,92 \\
\hline Cne & 628,24 & 2,51 & 10,00 & 0,18 & 0,10 & 0,91 & 3,60 \\
\hline Guareasp. & 195,60 & 0,78 & 50,00 & 0,89 & 0,20 & 1,82 & 3,49 \\
\hline Heliconiasp. & 590,70 & 2,36 & 10,00 & 0,18 & 0,10 & 0,91 & 3,45 \\
\hline Palicourea thyrsifiora & 59,77 & 0,24 & 00 & 1,79 & 0,10 & 0,91 & 2,93 \\
\hline Beilschmiediasp. & 378,90 & 1,51 & 20,00 & 0,36 & 0,10 & 0,91 & 2,78 \\
\hline$O C$ & 74 & 058 & 20,00 & 0,36 & 0,20 & 1,82 & 2,76 \\
\hline Mikania banisteriae & 35,78 & 0,14 & 90,00 & 1,61 & 0,10 & 0,91 & 2,66 \\
\hline Anthurium pedatum & 52,71 & 0,21 & 30,00 & 0,54 & 0,20 & 1,82 & 2,56 \\
\hline S & 10 & 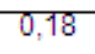 & 20,00 & 6 & 20 & 1,82 & 35 \\
\hline Trichanthera gigantea & 237,78 & 0,95 & 20,00 & 0,36 & 0,10 & 0,91 & 2,22 \\
\hline Mauria heterophylla & 147,41 & 0,59 & 30,00 & 0,54 & 0,10 & 0,91 & 2,03 \\
\hline MiC & 29 & 0 & 50,00 & 0,89 & 0,10 & 0,91 & 1, \\
\hline Mollinedia sp. & 59,51 & 0,24 & 20,00 & 0,36 & 0,10 & 0,91 & 1,50 \\
\hline Geonomajussieuana & 88,19 & 0,35 & 10,00 & 0,18 & 0,10 & 0,91 & 1,44 \\
\hline Myrsine coriacea & 87,10 & 0,35 & 10,00 & 0,18 & 0,10 & 0,91 & 1,44 \\
\hline Cridemia ciliata & 26,58 & 0,11 & 10,00 & 0,18 & 0,10 & 0,91 & 1,19 \\
\hline Solanum acerifolium & 13,28 & 0,05 & 10,00 & 0,18 & 0,10 & 0,91 & 1,14 \\
\hline
\end{tabular}

$D=$ densidad. $D R=$ densidad relativa. $F=$ Frecuencia. $F R=$ Frecuencia Relativa. $D o=$ Iominancia. DoR = Dominancia Relativa. IVI = Indice de Valor de Importancia. 
Para citar este artículo: López Vargas, L.E., Becoche Mosquera, J.M., Macías Pinto, D.J., Ruiz Montoya, K., Velasco Reyes, A. y Pineda, S. (2015). Estructura y composición florística de la Reserva Forestal - Institución Educativa Cajete, Popayán (Cauca). Revista Luna Azul, 41, 131-151. Recuperado de http://lunazul.ucaldas.edu.co/index.php?option=content\&tas k=view\&id=1059 